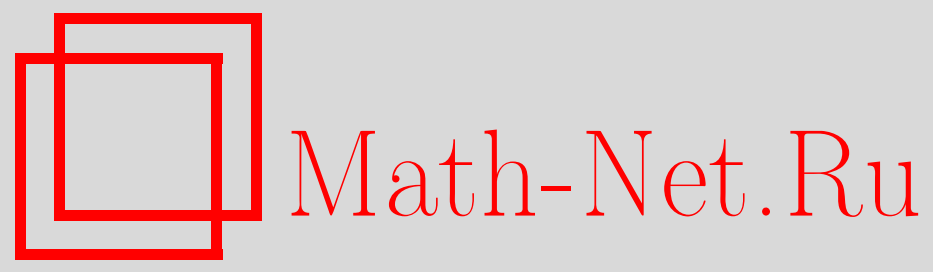

Ш. Р. Нурутдинов, Моделирование цепей Маркова в полях Галуа, Дискрет. матем., 2004, том 16, выпуск 2, 136-147

DOI: https://doi.org/10.4213/dm159

Использование Общероссийского математического портала Math-Net.Ru подразумевает, что вы прочитали и согласны с пользовательским соглашением http://www.mathnet.ru/rus/agreement

Параметры загрузки:

IP : 3.85 .73 .92

26 апреля 2023 г., 12:58:44

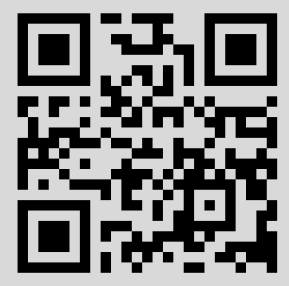




\title{
Моделирование цепей Маркова в полях Галуа
}

\author{
() 2004 г. Ш. Р. Нурутдинов
}

Автоматной моделью детерминированной функции является вероятностный автомат $A_{1}=\left(S, Y, P_{s}, \lambda(s)\right)$, где $S$ - множество состояний цепи Маркова, $P_{s}$ - стохастическая матрица размера $m_{1} \times m_{1}, Y-$ выходной алфавит мощности $m_{2} \leqslant m_{1}$. Автоматной моделью вероятностной функции является вероятностный автомат $A_{2}=\left(S, Y, P_{S}, P_{y}\right)$, где элементы $S, Y, P_{s}$ те же, что в $A_{1}$, а $P_{y}$ - стохастическая $m_{1} \times m_{2}$ матрица.

В настоящей работе решается задача синтеза генераторов конечных однородных цепей Маркова на основе аналитического аппарата полиномиальных функций над полем Галуа. Предложен способ вычисления коэффициентов полинома от нескольких переменных, моделирующего любое отображение поля Галуа в себя. Исследован случай моделирования любого конечного автомата однородной вычислительной структурой, определенной в поле Галуа, причем автоматные отображения реализуются как полиномиальные функции в поле Галуа. Галуа

В качестве базовых полиномов используются полиномиальные функции над полем

$$
f_{1}(x, s)=\sum_{i, j=0}^{r} a_{i j} x^{j} s^{i}, \quad f_{2}(s)=\sum_{i=0}^{r} b_{i} s^{i},
$$

где $r=2^{n}-1, x, s, b_{i}, a_{i j} \in G F\left(2^{n}\right)$.

Приведены представления автомата $A_{1}$ полиномиальной моделью над полем $G F\left(2^{n}\right)$ вида $M_{1}=\left(\hat{\mu}_{1}, f_{1}(x, s), f_{2}(s)\right)$, где $\hat{\mu}_{1}$ - случайная дискретная величина с множеством значений $\mu \in G F\left(2^{n}\right)$, задаваемая вектором вероятностей $\bar{P}=\left(p_{1}, \ldots, p_{k_{1}}\right)$ таким, что

$$
P_{s}=\sum_{i=1}^{k_{1}} p_{i} B_{i}
$$

где $B_{i}$ - стохастические булевы матрицы и $k_{1} \geqslant m_{1}^{2}-m_{1}+1$, и автомата $M_{2}=\left(\hat{\mu}_{1}, f_{1}(x, s), f_{2}(s), \hat{\mu}^{\prime}, f_{3}\left(x^{\prime}, s\right)\right)$, где $\hat{\mu}_{1}^{\prime}$ - случайная дискретная величина с множеством значений $\mu^{\prime} \in G F\left(2^{n}\right)$, и вектором вероятностей $\hat{P}=\left(p_{1}, \ldots, p_{k_{2}}\right)$ таким, что

$$
P_{y}=\sum_{i=1}^{k_{2}} p_{i} B_{i}
$$

где $B_{i}-$ стохастические булевы матрицы и $k_{2} \geqslant m_{1}^{2}-m_{1}+1$. Задача представления случайной дискретной величины над полем $G F\left(2^{n}\right)$ была решена ранее.

Работа выполнена при поддержке Российского фонда фундаментальных исследований, проект 99-01-00163. 


\section{1. Введение}

Цепи Маркова относятся к классу популярных случайных процессов для различных приложений. В связи с этим задаче генерации марковских последовательностей уделяется большое внимание. На основные принципиальные вопросы, связанные с синтезом генераторов цепей Маркова, ответы получены на базе теоретико-автоматных идей в ряде работ (см., например, [1-2]).

В полях Галуа эффективно реализуются системы потоковой обработки $n$-мерных двоичных векторов. Это открывает возможность моделирования рассматриваемых абстрактных структур устройствами, допускающими параллельную реализацию.

Решается задача синтеза автономных моделей марковских функций в базисе полиномов над полем $G F\left(2^{n}\right)$. Рассматриваются марковские функции двух типов: детерминированные функции, задаваемые над множеством состояний цепи Маркова путем укрупнения состояний, и вероятностные функции, задаваемые стохастической матрицей.

\section{2. Реализация отображения, заданного над элементами поля Галуа, многочленом от одной переменной}

Пусть $G=G F\left(2^{n}\right)$. Рассмотрим отображение $Y: G \rightarrow G$. Любое отображение конечного поля в себя можно задать многочленом $f$ над этим полем [3] вида

$$
f(x)=\sum_{i=0}^{r} a_{i} x^{i}, \quad r=2^{n}-1, \quad x, a_{i} \in G .
$$

Найдем коэффициенты многочлена (1) по заданному отображению $Y$. Для этого построим систему

$$
f(\alpha)=\sum_{i=0}^{r} a_{i} \alpha^{i}, \quad r=2^{n}-1,
$$

где $\alpha=0,1, \xi, \xi^{2}, \ldots, \xi^{2^{n}-2}, \xi$ - примитивный элемент $G$.

Матрица коэффициентов этой системы есть (с учетом свойств элементов поля)

$$
C=\left(\begin{array}{ccccccc}
1 & 0 & 0 & \ldots & 0 & \ldots & 0 \\
1 & 1 & 1 & \ldots & 1 & \ldots & 1 \\
1 & \xi & \xi^{2} & \ldots & \xi^{2^{n}-2} & \ldots & 1 \\
1 & \xi^{2} & \xi^{4} & \ldots & \xi^{2\left(2^{n}-2\right) \bmod \left(2^{n}-1\right)} & \ldots & 1 \\
\ldots \ldots \ldots \ldots \ldots \ldots \ldots \ldots \ldots \ldots & \ldots \ldots \ldots \ldots \ldots \ldots
\end{array}\right) .
$$

Систему (2) перепишем в матричном виде $F=C A$, где

$$
F=\left(f(0), f(1), f(\xi), \ldots, f\left(\xi^{2^{n}-2}\right)\right)^{T}, \quad A=\left(a_{0}, a_{1}, \ldots, a_{2^{n}-1}\right)^{T}
$$


Тогда $A=C^{-1} F$. Непосредственным подсчетом можно убедится, что

$$
C^{-1}=\left(\begin{array}{ccccccc}
1 & 0 & 0 & \ldots & 0 & \ldots & 0 \\
0 & 1 & \xi^{r-1} & \ldots & \xi^{(r-1) i \bmod (r)} & \ldots & \xi \\
\ldots \ldots \ldots & \ldots \ldots \ldots \ldots \ldots \ldots \ldots \ldots \ldots \ldots \ldots \ldots \ldots \ldots & \ldots \ldots \ldots \\
0 & 1 & \xi^{2} & \ldots & \xi^{2 i \bmod (r)} & \ldots & \xi^{r-2} \\
0 & 1 & \xi & \ldots & \xi^{i} & \ldots & \xi^{r-1} \\
1 & 1 & 1 & \ldots & 1 & \ldots & 1
\end{array}\right),
$$

где $r=2^{n}-1,1 \leqslant i \leqslant r-1$.

Теорема 1. Если $Y: G \rightarrow G-$ произвольная функиия, которая отображсает $G=G F\left(2^{n}\right)$ в $G$, то существует единственный многочлен

$$
f(x)=\sum_{i=0}^{r} a_{i} x^{i}, \quad r=2^{n}-1, \quad x, a_{i} \in G,
$$

являюшийся представлением отображения $G$ в том смысле, что $f(c)=Y(c)$ для всех $c \in G$. Коэффичиенты многочлена $f$ вычисляются по формуле

$$
A=C^{-1} F \text {, }
$$

где $A=\left(a_{0}, a_{1}, \ldots, a_{r}\right)^{T}$ - вектор коэффичиентов многочлена (1),

$$
F=\left(Y(0), Y(1), Y(\xi), \ldots, Y\left(\xi^{r-1}\right)\right)^{T},
$$

где $\xi$ - примитивный элемент поля $G$, есть вектор значений отображения $Y$ на элементах поля $G, C^{-1}-$ матрича (3), причем $C C^{-1}=E$, где $C$-матрича коэффичиентов cucmembl (2).

Пример 1. Пусть $G=G F\left(2^{2}\right)$. Выберем $m(x)=1+x+x^{2}$. Тогда $G=\left\{0,1, \xi, \xi^{2}\right\}$, где $\xi$ - примитивный элемент. На элементах поля $G$ определим отображение $Y$, переводящее точки $\left\{0,1, \xi, \xi^{2}\right\}$ соответственно в $\{\xi, 1,0,1\}$. Тогда многочлен, являющийся представлением отображения $Y$, имеет вид

$$
f(x)=\sum_{i=0}^{3} a_{i} x^{i}, \quad x_{i}, a_{i} \in G .
$$

Найдем коэффициенты многочлена $f(x)$. Значения отображения $Y$ на элементах поля $G$ образуют вектор $F=\left(Y(0), Y(1), Y(\xi), Y\left(\xi^{2}\right)\right)^{T}=(\xi, 1,0,1)^{T}$. Согласно (4), $A=C^{-1} F$, где $A=\left(a_{0}, a_{1}, a_{2}, a_{3}\right)^{T}$, a

$$
C^{-1}=\left(\begin{array}{cccc}
1 & 0 & 0 & 0 \\
0 & 1 & \xi^{2} & \xi \\
0 & 1 & \xi & \xi^{2} \\
1 & 1 & 1 & 1
\end{array}\right)
$$

Отсюда

$$
A=\left(\begin{array}{cccc}
1 & 0 & 0 & 0 \\
0 & 1 & \xi^{2} & \xi \\
0 & 1 & \xi & \xi^{2} \\
1 & 1 & 1 & 1
\end{array}\right)\left(\begin{array}{l}
\xi \\
1 \\
0 \\
1
\end{array}\right)=\left(\begin{array}{c}
\xi \\
\xi^{2} \\
\xi \\
\xi
\end{array}\right)
$$

Окончательно,

$$
f(x)=\xi+\xi^{2} x+\xi x^{2}+\xi x^{3}
$$




\section{3. Моделирование конечного автомата однородной сетью над $G F\left(2^{n}\right)$}

Работу цифрового устройства можно описать с помощью конечного автомата

$$
(X, Y, Q, \sigma, \lambda),
$$

где $X$ и $Y$ - входной и выходной алфавиты, $\sigma$ - функция переходов, а $\lambda-$ функщия выходов.

Пусть $M=(X, Y, Q, \sigma, \lambda)$ и $M_{1}=\left(X_{1}, Y_{1}, Q_{1}, \sigma_{1}, \lambda_{1}\right)$ - конечные автоматы.

Принято говорить, что автомат $M_{1}$ моделирует автомат $M$, если существуют отображения $\varphi: X \rightarrow X_{1}, \Psi: Y \rightarrow Y_{1}, \chi: Q \rightarrow Q_{1}$ такие, что

$$
\begin{aligned}
& \chi(\sigma(x, q))=\sigma_{1}(\varphi(x), \chi(q)), \\
& \Psi(\lambda(x, q))=\lambda_{1}(\varphi(x), \chi(q)),
\end{aligned}
$$

то есть множества $X, Y, Q$ можно отождествить с некоторыми подмножествами $X_{1}{ }^{\prime} \subset X_{1}$, $Y_{1}{ }^{\prime} \subset Y_{1}, Q_{1}{ }^{\prime} \subset Q_{1}$, при этом на указанных подмножествах автомат $M_{1}$ ведет себя так же, как и $M$ (входной алфавит автомата $M$ можно закодировать с помощью букв множества $X_{1}{ }^{\prime} \subset X_{1}$, а состояниям $Q$ ставятся в соответствие точки множества $Q_{1}{ }^{\prime} \subset Q_{1}$ ). После этого, находясь в состояниях множества $Q_{1}{ }^{\prime}$, под действием букв алфавита $X_{1}$ автомат $M_{1}$ ведет себя также, как и $M$.

Под однородной сетью будем понимать сеть автоматов [5]

$$
M_{i}=\left(X_{i}, Y_{i}, Q_{i}, \sigma_{i}, \lambda_{i}\right) \text {, }
$$

где $i=1, \ldots, N$, обмен информацией между которыми производится по однородным и локальным связям. Положим $Q^{\prime}=Q_{1} * Q_{2} * \ldots * Q_{N}$. Часть входов и выходов автоматов $M_{i}$, $1=1, \ldots, N$, являются входами и выходами сети. Пусть $X^{\prime}-$ входной алфавит и $Y^{\prime}-$ выходной алфавит сети. Функционирование сети описывает автомат $M^{\prime}=\left(X^{\prime}, Y^{\prime}, Q^{\prime}, \sigma^{\prime}, \lambda^{\prime}\right)$. Покажем, как по произвольному автомату $M=(X, Y, Q, \sigma, \lambda)$ можно построить однородную сеть, причем автомат $M^{\prime}$, описывающий сеть, будет моделировать работу автомата $M$. Рассмотрим случай, когда автомат $M$ является комбинационной схемой. Эта схема определяется отображением $\lambda: X \rightarrow Y$. Пусть $n$ - наименьшее из натуральных чисел, для которых выполнены неравенства $2^{n} \geqslant|X|, 2^{n} \geqslant|Y|$. Пусть $G=G F\left(2^{n}\right)$. Закодируем элементы множеств $X$ и $Y$ элементами поля $G$ (это эквивалентно построению отображений $\varphi$ и $\Psi$ в (5)). Будем считать, что отображение $\lambda$ определено на подмножестве $G$ со значениями в $G$. Зададим отображение $\lambda$ с помощью многочлена от одной переменной над полем $G$ :

$$
g(x)=\sum_{i=0}^{r} a_{i} x^{i}, \quad r=2^{n}-1, \quad x, a_{i} \in G .
$$

Такой многочлен можно построить с помощью теоремы 1. Таким образом, построен автомат $M^{\prime}$, моделирующий исходный автомат $M$. Выбрав одну из форм реализации многочлена (6) однородной вычислительной сетью [8], получим реализацию исходной комбинированной схемы в виде однородной вычислительной сети.

Пример 2. Пусть дана комбинационная схема, заданная системой булевых уравнений $y_{1}=x_{1} x_{3}, y_{2}=x_{3} x_{2}$. Закодируем элементы множеств $X=\left\{x: x=\left(x_{1} x_{2} x_{3}\right)\right\}$ и 
$Y=\left\{y: y=\left(y_{1} y_{2} 0\right)\right\}$ элементами поля $G F\left(2^{3}\right)$. Для того чтобы определить элементы поля $G F\left(2^{3}\right)$, выберем примитивный многочлен $p(x)=1+x+x^{3}$. Элементы поля $G F\left(2^{3}\right)$, представленные в виде степени примитивного элемента $\xi$, имеют следующее векторное представление:

$$
\begin{aligned}
0 \rightarrow(000), \quad 1 \rightarrow(100), \quad \xi \rightarrow(101), \quad \xi^{2} \rightarrow(111), \quad \xi^{3} \rightarrow(011), \\
\xi^{4} \rightarrow(110), \quad \xi^{5} \rightarrow(001), \quad \xi^{6} \rightarrow(010) .
\end{aligned}
$$

После прямого кодирования элементов множеств $X$ и $Y$, получим отображение

$$
\left(0,1, \xi, \xi^{2}, \xi^{3}, \xi^{4}, \xi^{5}, \xi^{6}\right) \rightarrow\left(0,0,1, \xi^{4}, \xi^{6}, 0,0,0\right) .
$$

Представим его в виде многочлена от одной переменной. Для этого воспользуемся теоремой 1.

В этом случае

$$
C^{-1}=\left(\begin{array}{cccccccc}
1 & 0 & 0 & 0 & 0 & 0 & 0 & 0 \\
0 & 1 & \xi^{6} & \xi^{5} & \xi^{4} & \xi^{3} & \xi^{2} & \xi \\
0 & 1 & \xi^{5} & \xi^{3} & \xi & \xi^{6} & \xi^{4} & \xi^{2} \\
0 & 1 & \xi^{4} & \xi & \xi^{5} & \xi^{2} & \xi^{6} & \xi^{3} \\
0 & 1 & \xi^{3} & \xi^{6} & \xi^{2} & \xi^{5} & \xi & \xi^{4} \\
0 & 1 & \xi^{2} & \xi^{4} & \xi^{6} & \xi & \xi^{3} & \xi^{5} \\
0 & 1 & \xi & \xi^{2} & \xi^{3} & \xi^{4} & \xi^{5} & \xi^{6} \\
1 & 1 & 1 & 1 & 1 & 1 & 1 & 1
\end{array}\right), \quad F=\left(0,0,1, \xi^{4}, \xi^{6}, 0,0,0\right)
$$

Коэффициенты многочлена от одной переменной, реализующего отображение (7), определяются с помощью выражения

$$
A=C^{-1} F=\left(\xi^{4}, \xi^{5}, \xi^{5}, \xi, \xi^{3}, 0,0\right) .
$$

Таким образом, получили многочлен

$$
g(x)=\xi^{4}+\xi^{5} x+\xi^{5} x^{2}+\xi x^{3}+\xi^{3} x^{4} .
$$

\section{4. Реализация функции от $s$ переменных в $G F\left(2^{n}\right)$ многочленом от $s$ переменных}

Лемма 1 ([4]). Пусть $G=G F\left(2^{n}\right), a \varphi: G^{s} \rightarrow G$ - произвольная функция от $s$ переменных, отображающая $G * G * \ldots * G$ в $G$, тогда существует единственный многочлен

$$
f\left(x_{1}, \ldots, x_{s}\right)=\sum_{i_{1}, \ldots, i_{s}=0}^{r} a_{i_{1}, \ldots, i_{s}} x_{1}^{i_{1}} x_{2}^{i_{2}} \ldots x_{s}^{i_{s}},
$$

где $r=2^{n}-1, a_{i_{1}, \ldots, i_{s}}, x_{1}, \ldots, x_{s} \in G$, являющийся представлением отображения $\varphi в$ том смысле, что $f(c, d, \ldots, e)=\varphi(c, d, \ldots, e)$ для всех $c, d, \ldots, e \in G$.

Найдем коэффициенты многочлена по заданному отображению $\varphi$. Для этого введем следующие обозначения. Пусть $Y$ - матрица в $G^{s}$, и пусть знак * означает, что соответствующий индекс пробегает все значения от верхней до нижней границы. Если при записи элемента матрицы заменить некоторый индекс знаком $*$, а остальные индексы зафиксировать, то получим вектор из элементов исходной матрицы. Назовем этот вектор сечением. 
Пример 3. Пусть задана матрица

$$
Y=\left(\begin{array}{ll}
Y_{11} & Y_{12} \\
Y_{21} & Y_{22}
\end{array}\right)
$$

Тогда $Y(*, 1)$ является вектором $\left(Y_{11}, Y_{21}\right)^{T} ; Y(*, 2)$ - вектором $\left(Y_{12}, Y_{22}\right)^{T} ; Y(2, *)-$ вектором $\left(Y_{21}, Y_{22}\right)^{T}$.

Теорема 2. Пусть $G=G F\left(2^{n}\right)$. Если $\varphi: G^{s} \rightarrow G-$ произвольная функиия от $s$ переменных, отображающая $G^{s}$ в $G$, то коэффичиенты многочлена $f$ в (8) могут быть вычислены с помощью системы

$$
\begin{aligned}
Y^{(1)}\left(*, i_{2}, \ldots, i_{s}\right) & =C^{-1} Y\left(*, i_{2}, \ldots, i_{s}\right), \quad i_{2}, \ldots, i_{s}=0,1, \ldots, r, \\
Y^{(2)}\left(i_{1}, *, \ldots, i_{s}\right) & =C^{-1} Y^{(1)}\left(i_{1}, *, \ldots, i_{s}\right), \quad i_{1}, i_{3}, i_{4}, \ldots, i_{s}=0,1, \ldots, r, \\
\ldots & \\
Y^{(s)}\left(i_{1}, \ldots, i_{s-1}, *\right) & =C^{-1} Y^{(s-1)}\left(i_{1}, \ldots, i_{s-1}, *\right), \quad i_{1}, i_{2}, \ldots, i_{s-1}=0,1, \ldots, r, \\
A & =Y^{(s)},
\end{aligned}
$$

где $Y$ - матрица порядка s из значений функции $\varphi$ на элементах поля $G$,

$$
Y_{0 \ldots, 0}=\varphi(0, \ldots, 0), \quad Y_{i_{1}, \ldots, i_{s}}=\varphi\left(\xi^{i_{1}-1}, \ldots, \xi^{i_{s}-1}\right),
$$

$\xi$ - примитивный элемент поля $G, i_{1}, \ldots, i_{s}=1, \ldots, r, r=2^{n}-1$. Матрича $C^{-1}$ была определена в (3). Матрича $A=\left\{a_{i_{1}, \ldots, i_{s}}\right\}, i_{1}, \ldots, i_{s}=0,1, \ldots, r$ содержит значения коэффичиентов многочлена (8).

Доказательство. В доказательстве теоремы используем правило вычисления коэффищиентов многочлена (1) с помощью выражения (4). Предположим, что система (9) верна для многочлена от $k$ переменных. Рассмотрим многочлен

$$
f\left(x_{1}, \ldots, x_{k+1}\right)=\sum_{i_{1}, \ldots, i_{k+1}=0}^{r} a_{i_{1} \ldots i_{k+1}} x_{1}{ }^{i_{1}} \ldots x_{k+1}{ }^{i_{k+1}} .
$$

Зафиксируем переменные $x_{1}, \ldots, x_{k}$, тогда многочлен (10) запишется в виде

$$
f\left(\dot{c}, d, \ldots, e, x_{k+1}\right)=\sum_{i_{k+1}=0}^{r} a_{i_{k+1}}(c, d, \ldots, e) x_{k+1}^{i_{k+1}} .
$$

Многочлен (11) является многочленом от одной переменной. Коэффициенты $a_{i_{k+1}}(c, d, \ldots, e), i_{k+1}=0,1, \ldots, r$, могут быть вычислены при помощи (4)

$$
B=C^{-1}\left(\varphi(c, d, \ldots, e, 0), \varphi(c, d, \ldots, e, 1), \varphi(c, d, \ldots, e, \xi), \ldots, \varphi\left(c, d, \ldots, e, \xi^{r-1}\right)\right)^{T}
$$

Получаем, что

$$
B=\left(a_{0}(c, d, \ldots, e), a_{1}(c, d, \ldots, e), \ldots, a_{r}(c, d, \ldots, e)\right)^{T} .
$$

При этом вектор $B$ в (12) является сечением $Y\left(i_{1}, \ldots, i_{k}, *\right)$, где матрица $Y$ содержит значения функция $\varphi\left(x_{1}, \ldots, x_{k+1}\right)$. Поэтому (12) можно записать в виде $B=C^{-1} Y\left(i_{1}, \ldots, i_{k}, *\right)$, где $i_{1}, \ldots, i_{k}$, фиксированы. Далее получим, что

$$
Y^{(1)}\left(i_{1}, \ldots, i_{k}, *\right)=C^{-1} Y\left(i_{1}, \ldots, i_{k}, *\right) .
$$


Коэффициенты $a_{i_{k}}\left(x_{1}, \ldots, x_{k}\right), i_{k}=0,1, \ldots, r$, в свою очередь являются функциями от $k$ переменных $x_{1}, \ldots, x_{k}$ и могут быть представлены многочленами, коэффициенты которых вычисляются согласно предположению для $k$ переменных. Значения функций $a_{i_{k}}\left(x_{1}, \ldots, x_{k}\right), i_{k}=0,1, \ldots, r$, на элементах поля $G$ находятся в соответствующих сечениях матрицы $Y$ и могут быть использованы при вычислениях по системе (9). Таким образом, в конце вычислений получим значения козффициентов многочлена (9), которые будут находиться в матрице $A$.

Следствие 1. При $s=2$ коэффичиенты многочлена (8) вычисляются по формуле

$$
A=C^{-1} Y\left(C^{-1}\right)^{T} \text {, }
$$

где $Y$ - матрииа 2-го порядка, содержащая значения функции ч на элементах поля $G$. Доказательство. При $s=2$ система (9) примет вид

$$
\begin{aligned}
Y^{(1)}\left(*, i_{2}\right) & =C^{-1} Y\left(*, i_{2}\right), & & i_{2}=0,1, \ldots, r, \\
Y^{(2)}\left(i_{1}, *\right) & =C^{-1} Y^{(1)}\left(i_{1}, *\right), & & i_{1}=0,1, \ldots, r, \\
A & =Y^{(2)} & &
\end{aligned}
$$

Значениями сечений $Y^{(1)}\left(*, i_{2}\right), i_{2}=0,1, \ldots, r$, являются столбцы матрицы $Y$, откуда следует, что первое выражение системы (14) совпадает с выражением $Y^{(1)}=C^{-1} Y$.

Значениями сечений $Y^{(2)}\left(i_{1}, *\right), i_{1}=0,1, \ldots, r$, являются строки матрицы $Y^{(1)}$, поэтому второе выражение системы (14) может быть записано в виде

$$
Y^{(2)}=C^{-1}\left(Y^{(1)}\right)^{T}=C^{-1}\left(C^{-1} Y\right)^{T}=C^{-1} Y^{T}\left(C^{-1}\right)^{T} .
$$

Следует заметить, что матрица $Y^{(2)}$ транспонирована относительно расположения переменных отображения. Окончательно,

$$
A=Y^{(2)^{T}}=C^{-1} Y\left(C^{-1}\right)^{T} .
$$

Пример 4. Пусть $G=G F\left(2^{2}\right)$ и значения функции $\varphi: G * G \rightarrow G$ заданы матрицей

$$
Y=\left(\begin{array}{cccc}
1 & 1 & 1 & 0 \\
\xi^{2} & 1 & \xi^{2} & 0 \\
\xi^{2} & 0 & 1 & 0 \\
0 & 0 & \xi^{2} & \xi^{2}
\end{array}\right),
$$

где $\xi$ - примитивный элемент, являющийся корнем многочлена $m(x)=1+x+x^{2}$. Реализуем функцию $\varphi$ многочленом от двух переменных

$$
f(x, y)=\sum_{i, j=0}^{3} a_{i j} x^{i} y^{j}, \quad x, y, a_{i j} \in G .
$$

Используя (13), найдем коэффициенты $a_{i j}$. Матрица $C^{-1}$ имеет вид

$$
C^{-1}=\left(\begin{array}{cccc}
1 & 0 & 0 & 0 \\
0 & 1 & \xi^{2} & \xi \\
0 & 1 & \xi & \xi^{2} \\
1 & 1 & 1 & 1
\end{array}\right)
$$


Таблица 1.

\begin{tabular}{l|llll} 
& $x_{1}$ & $x_{2}$ & $x_{3}$ & $x_{4}$ \\
\hline$q_{1}$ & $q_{1}$ & $q_{1}$ & $q_{1}$ & $q_{2}$ \\
$q_{2}$ & $q_{2}$ & $q_{2}$ & $q_{3}$ & $q_{3}$ \\
$q_{3}$ & $q_{4}$ & $q_{3}$ & $q_{1}$ & $q_{4}$ \\
$q_{4}$ & $q_{4}$ & $q_{4}$ & $q_{2}$ & $q_{1}$
\end{tabular}

Тогда

$$
A=C^{-1} Y\left(C^{-1}\right)^{T}=\left(\begin{array}{cccc}
1 & 1 & \xi & 1 \\
\xi & 0 & 0 & 1 \\
\xi^{2} & 0 & 1 & \xi \\
1 & 0 & \xi & \xi
\end{array}\right)
$$

и $f(x, y)=1+y+\xi y^{2}+y^{s}+x\left(\xi+y^{s}\right)+x^{2}\left(\xi^{2}+y^{2}+\xi y^{s}\right)+x^{s}\left(1+\xi^{2}+\xi y^{s}\right)$.

\section{5. Моделирование автомата с памятью}

Опишем случай автомата без выходов. Такой автомат описывается тройкой $M=(X, Q, \delta(x, q))$. Выберем (см. [5]) наименьшее $n$ так, чтобы выполнялись неравенства $2^{n} \geqslant|X|, 2^{n} \geqslant|Q|$. Элементы множеств $X$ и $Q$ закодируем элементами поля $G=G F\left(2^{n}\right)$. Получим, что отображение $\delta(x, q)$ определено на подмножестве $G \times G$ со значениями в $G$. Представим его в виде многочлена

$$
f(x, q)=\sum_{i, j=0}^{r} a_{i j} x^{j} q^{i}, \quad r=2^{n}-1, \quad x, q, a_{i j} \in G .
$$

Тогда автомат $M$ моделируется автоматом $M^{\prime}=(G, G, f(x, q))$. Коэффициенты многочлена (15) вычисляются по формуле

$$
A=C^{-1} Y\left(C^{-1}\right)^{T}
$$

где $Y$ - матрица, содержащая значения функции $\delta(x, q)$ на элементах поля $G$.

Пример 5. Пусть дан автомат с памятью без выходов, заданный таблицей (см. таблицу 1), в которой каждой паре (входной сигнал- текущее состояние) ставится в соответствие новое состояние. Входной алфавит $X=\left\{x_{1}, x_{2}, x_{3}, x_{4}\right\}$, алфавит внутренних состояний $Q=\left\{q_{1}, q_{2}, q_{3}, q_{4}\right\}$. Элементы множеств $X$ и $Q$ закодируем элементами поля $G=G F\left(2^{2}\right)$ произвольным образом: $x_{1}=0, x_{2}=1, x_{3}=\xi, x_{4}=\xi^{2}, q_{1}=0, q_{2}=1$, $q_{3}=\xi, q_{4}=\xi^{2}$. Элементы поля $G$ зададим при помощи примитивного многочлена $m(x)=1+x+x^{2}$. Отношение $\delta(x, q)$, определенное на подмножестве $G \times G$, со значениями в $G$ представим в виде многочлена от двух переменных, аналогичного многочлену (15). Вычислим коэффициенты многочлена. Матрица $F$ и $C^{-1}$ имеют вид

$$
F=\left(\begin{array}{cccc}
0 & 1 & \xi^{2} & \xi^{2} \\
0 & 1 & \xi & \xi^{2} \\
0 & \xi & 0 & 1 \\
1 & \xi & \xi^{2} & 0
\end{array}\right), \quad C^{-1}=\left(\begin{array}{cccc}
1 & 0 & 0 & 0 \\
0 & 1 & \xi^{2} & \xi \\
0 & 1 & \xi & \xi^{2} \\
1 & 1 & 1 & 1
\end{array}\right)
$$


Коэффициенты многочлена от двух переменных вычислим согпасно выражению (16). Поэтому для матрицы коэффициентов многочлена $\delta(x, q)$ справедливы равенства

$$
\begin{aligned}
A=C^{-1} F\left(C^{-1}\right)^{T} & =\left(\begin{array}{cccc}
1 & 0 & 0 & 0 \\
0 & 1 & \xi^{2} & \xi \\
0 & 1 & \xi & \xi^{2} \\
1 & 1 & 1 & 1
\end{array}\right)\left(\begin{array}{llll}
0 & 1 & \xi^{2} & \xi^{2} \\
0 & 1 & \xi & \xi^{2} \\
0 & \xi & 0 & 1 \\
1 & \xi & \xi^{2} & 0
\end{array}\right)\left(\begin{array}{cccc}
1 & 0 & 0 & 1 \\
0 & 1 & 1 & 1 \\
0 & \xi^{2} & \xi & 1 \\
0 & \xi & \xi^{2} & 1
\end{array}\right) \\
& =\left(\begin{array}{cccc}
0 & \xi & \xi^{2} & 1 \\
\xi & 1 & 1 & \xi^{2} \\
\xi & \xi^{0} & 0 & 0 \\
1 & \xi & 1 & \xi
\end{array}\right)
\end{aligned}
$$

и

$$
\delta(x, q)=\xi x+\xi^{2} x^{2}+x^{3}+\left(\xi+x+x^{2}+\xi^{2} x^{3}\right) q+(\xi+\xi x) q^{2}+\left(1+\xi x+x^{2}+\xi x^{3}\right) q^{3} .
$$

\section{6. Полиномиальная модель цепи Маркова}

Зададим конечную однородную цепь Маркова системой

$$
P_{(m)}=\left(S, P, \pi_{0}\right)
$$

где $S=\left(s_{1}, \ldots, s_{m}\right)$ - множество состояний цепи Маркова, $P$ - стохастическая матрица размера $m \times m, \pi_{0}-m$-мерный стохастический вектор, определяющий начальное распределение вероятностей состояний цепи Маркова.

В соответствии с $[1,2]$ будем называть простыми стохастические матрицы, элементы которых равны нулю или единице, при этом матрица имеет в каждой строке одну и только одну единицу.

Обозначим символом $\hat{\mu}$ дискретную случайную величину вида

$$
\hat{\mu}=\left(\begin{array}{cccc}
\mu_{1}, & \mu_{2}, & \ldots, & \mu_{l} \\
p_{1}, & p_{2}, & \ldots, & p_{l}
\end{array}\right),
$$

где $\mu_{i}, i=1, \ldots, l,-$ значения $\hat{\mu}, p_{i}$ - вероятности появления $\mu_{i}, i=1, \ldots, l, 0 \leqslant p_{i} \leqslant 1$, $p_{1}+\ldots+p_{l}=1$, а стохастический вектор $\left(p_{1}, \ldots, p_{l}\right)$ обозначим символом $\bar{P}$. На величину $l$ наложим ограничение $l \leqslant m^{2}-m+1$, связанное с размером матрицы $P$. Зададим конечное поле $G=G F\left(2^{n}\right)$ и полином от двух переменных над этим полем (см. $[4,7])$

$$
f(x, q)=\sum_{i, j=0}^{r} a_{i j} x^{j} q^{i}, \quad r=2^{n}-1
$$

$x, q, a_{i j} \in G, n$ определяется условием $l \leqslant 2^{n}-1$.

Справедлива следующая теорема синтеза.

Теорема 3. Для заданной системы (17) можно указать случайную величину $\hat{\mu}$ и полином $f(x, q)$ степени $r \geqslant l$ со случайным начальным значением переменной $q$ и коэффичиентами $a_{i j} \in G$ такими, что $\hat{\mu}$ может быть преобразована полиномиальной функцией $f(x, q)$ в заданную чепь Маркова. 
Для доказательства теоремы 3 нам потребуется следующая лемма.

Лемма 2 ([1]). Каждая стохастическая матрица $P$ размера $m \times m$ может быть представлена в виде стохастической линейной комбиначии не более чем $m^{2}-m+1$ простых матрич. Последовательность коэффичиентов любого из таких разложений определяет стохастический вектор $\bar{P}$, на основе которого можно однозначно получить все элементы заданной матрицы $P$.

Представим матрицу $P$ размера $m$ линейно стохастической комбинацией (см. [2])

$$
P=\sum_{k=1}^{l} p_{k} M\left(\mu_{k}\right),
$$

где $l \leqslant m^{2}-m+1, M\left(\mu_{k}\right)$ - простая матрица размера $m \times m$, соответствующая значению $\mu_{k}, 0 \leqslant p_{i} \leqslant 1, p_{1}+\ldots+p_{l}=1$.

В разложении (19) коэффициенты $p_{k}, k=1, \ldots, l$, задают искомый стохастический вектор $\bar{P}$, а множество простых матриц $M\left(\mu_{k}\right), k=1, \ldots, l$, однозначно задает функцию переходов $\delta(\mu, s)$ конечного автомата вида

$$
M=(\bar{\mu}, S, \delta(\mu, s))
$$

где $\bar{\mu}$ - входной алфавит, совпадающий с множеством значений случайной величины $\hat{\mu}, S$ - множество состояний автомата, совпадающее с множеством состояний системы (17). Элементы матриц $M\left(\mu_{k}\right)$, которые обозначим через $\pi_{i j}\left(\mu_{k}\right), i, j=1, \ldots, m$, задают функцию $\delta(\mu, s)$ следующим образом.

Если в $M\left(\mu_{k}\right)$ элемент $\pi_{i j}\left(\mu_{k}\right)=1$, то $\delta\left(\mu_{k}, s_{i}\right)=s_{j}, i, j=1, \ldots, m$.

Автомат (20) обладает следующим свойством (см. [1]): если на вход автомата $M$ подана последовательность независимых копий случайной величины $\hat{\mu}$, то последовательность его состояний является цепью Маркова.

Представим функцию $\delta(\mu, s)$ автомата (20) полиномом $f(x, q)$.

Нам потребуется следующая лемма.

Лемма 3. Пусть $G=G F\left(2^{n}\right), a \varphi: G \times G \rightarrow G$ - произвольная функция от двух переменных, отображающая $G \times G$ в $G$, тогда существует единственный полином вида (18), являющийся представлением отображения $\varphi$ в том смысле, что $f(c, d)=\varphi(c, d)$ для всех $c, d \in G \times G$.

Коэффициенты многочлена (18), представляющего заданное отображение $\varphi$, найдем, как в (13),

$$
A=C^{-1} Y\left(C^{-1}\right)^{T}
$$

где $A=\left\|a_{i j}\right\|, i, j=0,1, \ldots, r-1$, - матрица коэффициентов многочлена (18), $Y=\left\|y_{i j}\right\|$, $i, j=0,1, \ldots, r-1,-$ матрица значений отображения $\varphi$ на элементах поля $G$, матрица $C^{-1}$ построена как в (3).

Реализацию $\delta(\mu, s)$ полиномом (18) выполним, как в разделе 5. Выберем наименьшее $n$ так, чтобы выполнялись неравенства $2^{n} \geqslant|\bar{\mu}|, 2^{n} \geqslant|S|$, где $|A|$ - мощность множества $A$. Элементы множеств $\bar{\mu}$ и $S$ закодируем элементами поля $G$. Получим, что отображение $\delta(\mu, s)$ определено на подмножестве $G \times G$ со значениями в $G$ и оно может быть представлено полиномом (18). 
Таким образом, задание цепи Маркова в виде системы

$$
\left(\hat{\mu}, f(x, q), \pi_{0}\right)
$$

эквивалентно заданию системы (17).

Теорема 1 доказана.

На основе лемм 1 и 2 и соотношения (21) нетрудно показать, что для системы (22) справедливо и обратное теореме 1 утверждение, теорема анализа.

Теорема 4. Пусть заданы

$$
f(x, q)=\sum_{i, j=0}^{r} a_{i j} x^{j} q^{i}, \quad r=2^{n}-1, \quad a_{i j}, x, q \in G,
$$

смножеством $\left\{x_{i}\right\}, i=1, \ldots, l$, значений переменной х имножеством $\left\{q_{j}\right\}, j=1, \ldots, m$, значений переменной $q$, случайная величина $\hat{\mu}$ с множеством значений $\left\{\mu_{i}\right\} \subset\left\{x_{i}\right\}$ и со стохастическим вектором $\bar{P}$ и вектор $\pi_{0}$.

Тогда последовательность вычисленных значений полинома $f(x, q)$ является реализачией простой однородной чепи Маркова с множеством состояний $\left\{q_{j}\right\}$, описываемой стохастической матричей $P$ размера $\left|\left\{q_{j}\right\}\right|$, элементы которой однозначно определяются вектором $\bar{P}$ и полиномом $f(x, q)$.

Как одно из решений, рассмотрим реализацию отображения $\varphi: G \times G \rightarrow G$ при помощи многочлена от одной переменной. Решение основано на сведении многочлена вида (18) к многочлену от одной переменной, но над полем большей размерности. Полином $f(x, q)$ представляется в виде отображения $\delta(z): G F\left(2^{p}\right) \rightarrow G F\left(2^{p}\right)$, где $p=2 n$. При этом

$$
\delta(z)=z^{\prime}, \quad z, z^{\prime} \in G F\left(2^{p}\right), \quad z=(x, q)^{T} .
$$

$\delta$ может быть представлена полиномом от одной переменной

$$
\delta(z)=\sum_{i=0}^{s} a_{i} z^{i}, \quad s=2^{p}-1, \quad z, a_{i} \in G F\left(2^{p}\right) .
$$

Отметим, что при сведении полинома от двух переменных (18) к полиному от одной переменной, возникает задача минимизации количества слагаемых в сумме (24). Эта задача решена в [10].

Рассмотрим различные возможности генерации цепей Маркова, вытекающие из теорем 1 и 2:

(1) если модель (22) задана на основе системы (17), то последовательность значений $q$ есть цепь Маркова с законом, описываемым заданной стохастической матрицей $P$;

(2) если модель (22) задана произвольно, в соответствии с ограничениями, определяемыми теоремой 2 , то последовательность значений $q$ есть цепь Маркова, закон которой однозначно определяется по исходным данным (величине $\hat{\mu}$ и полиному $\delta(z))$;

(3) используя в модели (22) в качестве исходных данных различные стохастические векторы $\hat{P}$ или меняя коэффициенты $a_{i}, i=0,1, \ldots, s$, полинома $\delta(z)$, или меняя одновременно стохастический вектор и коэффициенты полинома $\delta(z)$, можно получать различные семейства простых однородных цепей Маркова. 
Задача полиномиального представления генератора случайной величины $\hat{\mu}$ в поле Галуа решена в [6], где показано, что структурная модель генератора случайной величины, построенная в классе комбинационных схем, может быть представлена некоторой полиномиальной функщией $f_{\hat{\mu}}$ над полем Галуа. Учитывая этот результат, процесс получения цепи Маркова можно рассматривать как суперпозищию преобразований, выполняемых полиномиальными функциями $f_{\hat{\mu}}$ и $\delta(z)$. Таким образом, система (17) может быть реализована суперпозицией вида $\delta(z) f_{\hat{\mu}}$ или $f(x, q) f_{\hat{\mu}}$ (если использовать полином $f(x, q)$ ), где начальное значение переменной $q$ выбирается в соответствии с заданным стохастическим вектором $\pi_{0}$.

Сложность реализации структурной модели (22) зависит от способа структурного представления полиномов над полем $G$. В [8-9] получены верхние оценки сложности реализации полиномиальной функции в случаях представления полиномиальной функция вида $f(x, q)$ параллельной, систолической, систолической векторной и последовательностной структурами.

\section{Список литературы}

1. Поспелов Д. А., Вероятностные автоматы. Энергия, Москва, 1970.

2. Бухараев Р. Г., Захаров В. М., Управляемые генераторы случайных кодов. Изд-во Казанского ун-та, Казань, 1978.

3. Лидл Р., Нидеррайтер Г., Конечные поля. т. 1, 2. Мир, Москва, 1988.

4. Нурутдинов Ш. Р., Реализация комбинационной схемы при помощи многочлена от нескольких переменных над конечным полем. Тезисы докладов VIII Всесоюзной конферениии по кибернетике, ч. 2. Горький, 1988, с. 61-62.

5. Нурутдинов Ш. Р., Столов Е. Л., Реализация автомата асинхронной сетью. Кибернетика (1988) №6, 108-109.

6. Захаров В. М., Нурутдинов Ш. Р., Шалагин С. В., Синтез автономных вероятностных автоматов на основе полей Галуа. В кн.: Исследования по информатике. Отечество, Казань, 2000, с. 107116.

7. Nurutdinov Sh. R., Markov chains in Galois fields. Intern. Conf. OFEA'2001. St. Peterburg, 47-49.

8. Захаров В. М., Нурутдинов Ш. Р., Шалагин С. В., Полиномиальное представление цепей Маркова над полем Галуа. Вестник Казанского гос. техн. ун-та (2001) №3, 27-31.

9. Захаров В. М., Нурутдинов Ш. Р., Шалагин С. В., Построение модели умножителя в полях Галуа. Материалы VII Международного семинара "Дискретная математика и ее приложения", ч. I. Изд-во центра прикладных исследований при механико-математическом факультете МГУ, Москва, 2001, с. 62-65.

10. Нурутдинов Ш. Р., Шалагин С. В., Минимизация количества элементов однородной вычислительной структуры. В кн.: Исследования по информатике. Отечество, Казань, 2000, с. 117-124. 